\title{
Percepción del riesgo en viajes compartidos. Efectos de la «Ubereconomía» en el transporte de taxis
}

DOI: https://doi.org/10.21158/01208160.n90.2021.2875

\author{
Solon Bevilacqua ${ }^{1}$ \\ Universidad Federal de Goias \\ solon@ufg.br \\ John Edward Neira-Villena² \\ Universidad Federal de Goias \\ johnneirav@ufg.br
}

Fecha de recepción: 02 de septiembre de 2019

Fecha de aprobación: 22 de marzo de 2021

Fecha de publicación: 11 de mayo de 2021

Cómo citar este artículo / To reference this article / Comment citer cet article / Para citar este artigo:

Bevilacqua, S.; Neira-Villena, J.E. (2021). Percepción del riesgo en viajes compartidos. Efectos de la «Ubereconomía» en el transporte de taxis. Revista Escuela de Administración de Negocios, (90), 213-232. DOI: https://doi.org/10.21158/01208160.n90.2021.2875

\section{Resumen}

En este estudio se investiga la percepción del riesgo y el contagio en red en el consumo de viajes compartidos - Uber y Carpooling-. El universo del estudio se relaciona directamente con los mercados impactados por la «Ubereconomía», principalmente, los taxis y los demás medios de transporte urbano. La hipótesis central de este estudio propone que los riesgos se diseminan a partir de una relación interpersonal entre participantes de una comunidad y el hecho de que ese riesgo puede ser propagado por un primer actor. La brecha explorada en este estudio contempla el contagio de la percepción del riesgo en economías de acceso para viajes compartidos y su impacto en el mercado de taxis. Los descubrimientos de la investigación indican que la seguridad y el confort son los principales aspectos del consumo de viajes compartidos. También se indican fallas en el sistema de evaluación de los usuarios y de los prestadores de servicio al realizar la validación del perfil a partir de redes sociales como Facebook y otros. El análisis permite concluir que, dado que la seguridad y la comodidad son atributos clave, cabe desarrollar funciones en las plataformas que tengan como objetivo mejorar los aplicativos, principalmente, en la de evaluación de los involucrados y en los indicadores de mantenimiento de los vehículos. Finalmente se establece la posibilidad extender los hallazgos de esta investigación para los otros negocios realizados en plataformas digitales de economía compartida.

Palabras clave: economía compartida; transporte urbano; percepción del riesgo; contagio en red; plataformas digitales; Uber; Carpooling.

\footnotetext{
1 Doctorado en Psicología - Pontifícia Universidade Católica, Brasil. Maestría en Administración - Universidade Federal de Uberlândia, Brasil. Graduación en Administración - Universidade Federal do Rio Grande do Sul, Brasil. ORCID: https://orcid. org/0000-0002-0050-3527

2 Doctorado en Ingeniería de Materiales - Universidade Federal do Rio Grande do Norte, Brasil. Maestría en Ingeniería Mecánica Universidade Federal do Rio Grande do Norte, Brasil. Graduación en Ingeniería Mecánica - Universidad Católica de Santa María, Perú. ORCID: https://orcid.org/0000-0003-2281-6505
} 


\title{
Perception of the risk in ridesharing. Effects of the $\ll$ Ubereconomy $\gg$ on cab transportation
}

\begin{abstract}
This study investigates risk perception and network contagion in ridesharing consumption -Uber and Carpooling-. The universe of the study is directly related to the markets that have been impacted by the «Ubereconomy», mainly cabs and other means of urban transportation. The central hypothesis of this study proposes that risks spread from an interpersonal relationship between participants in a community and the fact that this risk can be propagated by a first actor. The gap that has been explored in this study contemplates the contagion of risk perception in ridesharing access economies and its impact on the cab market. The research findings indicate that safety and comfort are the main aspects of ridesharing consumption. It also indicates flaws in the system for the evaluation of users and service providers when performing profile validation based on social networks such as Facebook and others. The analysis leads to the conclusion that, given that safety and comfort are key attributes, it is necessary to develop functions in the platforms aimed at improving the applications, mainly in the evaluation of those who are involved and in the indicators of vehicle maintenance. Finally, we establish the possibility of extending the findings of this research to other businesses that are carried out on digital sharing economy platforms.
\end{abstract}

Keywords: sharing economy; urban transportation; risk perception; network contagion; digital platforms; Uber; Carpooling.

\section{Percepção do risco em viagens compartilhadas. Efeitos da «Ubereconomia» no transporte de táxis}

\section{Resumo}

Este estudo pesquisa a percepção do risco e do contágio na rede do consumo de viagens compartilhadas - Uber e Carpooling- - O universo do estudo está diretamente relacionado com os mercados impactados pela «Ubereconomia», principalmente, táxis e outros meios de transporte urbano. A hipótese central deste estudo propõe que os riscos são disseminados a partir de uma relação interpessoal entre os participantes de uma comunidade e o fato de que esse risco pode ser propagado por um primeiro agente. A lacuna explorada neste estudo inclui o contágio da percepção do risco nas economias de acesso para viagens compartilhadas e seu impacto no mercado de táxis. Os resultados da pesquisa indicam que a segurança e o conforto são os principais aspectos do consumo de viagens compartilhadas. Também foram identificadas falhas no sistema de avaliação dos usuários e dos prestadores de serviço no momento da validação do perfil a partir de redes sociais como Facebook e outras. A análise permite concluir que, sendo segurança e conforto atributos essenciais, é possível desenvolver funções nas plataformas que tenham como objetivo melhorar as aplicações, principalmente na avaliação dos envolvidos e nos indicadores de manutenção dos veículos. Finalmente, estabelece-se a possibilidade de estender os descobrimentos desta pesquisa aos demais negócios realizados em plataformas digitais de economia compartilhada.

Palavras-chave: economia compartilhada; transporte urbano; percepção de risco; contágio em rede; plataformas digitais; Uber; Carpooling. 


\section{Perception du risque dans le covoiturage:} effets de 1 ' «uberéconomie » sur le transport urbain en taxi

\section{Résumé}

Cette étude examine la perception du risque et de la sécurité des réseaux dans la consommation de trajets de covoiturage de type Uber ou Carpooling. L'univers de l'étude est directement lié aux marchés impactés par $l^{\prime} \ll u b e r e ́ c o n o m i e »$, principalement les taxis et autres moyens de transports urbains. L'hypothèse centrale de cette étude propose que les risques soient disséminés à partir d'une relation interpersonnelle entre les participants d'une communauté et que ce risque puisse être propagé par un acteur initial. La problématique exposée ici comprend la contagion de la perception du risque dans les économies d'accès aux trajets partagés et son impact sur le marché du transport en taxis. Les résultats de l'étude indiquent que la sécurité et le confort sont les principaux aspects de la consommation du mode de transport en covoiturage. Les problèmes d'évaluation de la part des utilisateurs et des prestataires de services sont également signalés lors de la validation du profil à partir des réseaux sociaux de type Facebook. L'étude conclut que la sécurité et le confort sont des points clés du service, qu'il est fondamental de développer des fonctionnalités visant à améliorer les plateformes et les applications, surtout en ce qui concerne l'évaluation des acteurs et les indicateurs d'entretien des véhicules. Enfin, les résultats de cette étude peuvent être appliqués à d’autres entreprises et plateformes numériques de l'économie digitale.

Mots-clés: économie partagée; transport urbain; perception du risque; contagion du réseau; plates-formes numériques; Uber; covoiturage. 


\section{Introducción}

$\mathrm{L}$ a percepción de un riesgo está relacionada con mecanismos cognitivos individuales en los que los individuos colectan, procesan y forman percepciones como unidades desconectadas de un sistema social. Sin embargo, la visión clásica no ayuda a explicar cómo la percepción del riesgo puede variar entre grupos o dentro de una única comunidad. A partir de esa idea se origina el abordaje alternativo basado en la teoría de contagio en red. Este abordaje sugiere que los aspectos relacionales de los individuos y los sistemas autoorganizados construyen «grupos o comunidades de individuos con ideas similares» (Scherer y Cho, 2003), y así pueden contagiarse por los mismos riesgos.

En este estudio se investiga la percepción del riesgo y el contagio en red en el consumo de viajes compartidos. Sus efectos se perciben en el transporte público urbano, en especial en los taxis.

La teoría del contagio de riesgo será aplicada para comunidades de usuarios de viajes compartidos - Uber y Carpooling - El uso de plataformas de acceso para consumo compartido, como, por ejemplo, los pioneros Uber, AirBnB o BlaBlaCar, unió usuarios en comunidades que poseen el mismo interés y evalúan miembros de la red social a partir de notas puestas en un aplicativo. La práctica de dar una nota para un servicio realizado crea un enlace que une a todos los miembros en una comunidad de consumo que contempla temporalidad, anonimato, mediación de mercado, participación del consumidor, aplicativo y consumo comprometido (Bardhi y Eckhardt, 2012).

Estas unidades sociales se comportan como evaluadores de miembros consumidores y proveedores de servicios de esa comunidad, alimentando la idea de contagio en red. Por tanto, comunidades virtuales como las previstas en las economías compartidas atribuyen notas subjetivas para perfiles después de sus experiencias de consumo. Se trata de una cues- tión más próxima a un concepto de fuga de la economía tradicional, en el que prevalece la posesión y no la propiedad en el consumo de experiencias (Marcoux, 2009).

La hipótesis central de este estudio propone que los riesgos se diseminan a partir de una relación interpersonal entre participantes de una comunidad, y que ese riesgo puede propagarlo un primer actor (Scherer y Cho, 2003). Se asume que el riesgo es una consecuencia directa de los peligros inherentes a la situación física, mientras que actitudes en relación con el riesgo dependen de personalidades individuales (Douglas y Wildavsky, 1982). Se estudian tres hipótesis adyacentes al problema: ¿los riesgos percibidos por los usuarios de viajes compartidos son semejantes para sus variaciones?, ¿los individuos son contagiados por el riesgo cuando son insertados en la misma comunidad de consumo?, y, zlas variables manifiestas afectan la percepción del riesgo con la misma intensidad?

La brecha explorada en este estudio contempla el contagio de la percepción del riesgo y economías de acceso para viajes compartidos. El fenómeno ya fue investigado a partir de algunos puntos de vista intrapsíquicos importantes, pero el énfasis recae en estudios teóricos y empíricos que investigaron la percepción de riesgo en grupos sociales (Joffe, 2003; Kasperson et al., 1988; Masuda y Garvin, 2006; Murphy y Paté-Cornell, 1996; Paté-Cornell, 2012; Slovic, 1987). Se destaca un abordaje que entiende la selección de los riesgos interconectada a las formas sociales, en el cual la selección de los riesgos y la selección de cómo vivir se realizan en conjunto (Douglas y Wildavsky, 1982).

Vale la pena indicar que el tema de percepción de riesgo asociado a viajes compartidos es oportuno, ya que se trata de un asunto ampliamente estudiado en la literatura. El universo de los viajes realizados en taxis fue alterado profundamente. 
Viajar de forma compartida es la segunda manera más popular de desplazamiento, y tal vez una de las menos entendida (Guidotti et al., 2017).

A continuación, el contagio y la percepción del riesgo se detallan. Se describe la metodología y los análisis, las conclusiones, las contribuciones gerenciales y se presenta una agenda de estudios futuros.

\section{Redes sociales, contagio y percepción del riesgo (PR)}

$\mathrm{L}$ a capacidad de sentir y evitar las adversidades del ambiente es una condición para la supervivencia de todos los seres vivos. En el contexto de confianza, contagio y comunidad, los individuos aprenden con experiencias pasadas y provocan alteraciones ambientales. El resultado es la reducción de PR ambientales (Slovic, 1987). La habilidad de percibir riesgos se desarrolla bajo la influencia de culturas, lugares y experiencias colectivas (Douglas y Wildavsky, 1982; Kasperson et al., 1988; Masuda y Garvin, 2006), influenciada también por la presencia de emociones y simbolismos (Joffe, 2003). Por tanto, la perspectiva de comprender y responder a riesgos es efectiva cuando se obtienen respuestas de múltiples agentes - ¿qué debemos hacer? - en vez de atender a una perspectiva de un único tomador de decisión —iqué debo hacer? - (Cox, 2012).

Cuando un individuo juzga cuáles peligros deben ser temidos, cuáles riesgos valen la pena y quién debe enfrentarlos, estará enfrentando dilemas construidos a partir de sus vivencias en grupo. La mayoría de las personas no puede ser conscientes de la mayoría de los peligros la mayor parte de las veces. De esa manera, es difícil calcular con precisión la PR total a ser enfrentada.

¿Cómo las personas escogen los riesgos que deben ser aceptados y cuáles deben ser ignorados? La selección de los riesgos depende de las formas sociales seleccionadas, porque la decisión de cómo vivir se toma en conjunto. Valores comunes llevan a miedos comunes y a un acuerdo único de no temer otras cosas; al final toda sociedad depende de combinaciones de confianza y miedo (Douglas y Wildavsky, 1982). Se argumenta que la PR y su contagio se construyen a partir de experiencias vivenciadas en cada cultura de consumo.

Los orígenes para la teoría de contagio en red se remontan al año 1913, cuando estudiosos franceses iniciaron una serie de estudios que investigaron las diferencias entre los comportamientos aislados y en grupo. Ringelmann solicitó a individuos que jalasen una cuerda. Mientras más personas eran adicionadas a la actividad, el esfuerzo individual disminuía. La suma del esfuerzo conjunto, lógicamente, debería ser la suma de los esfuerzos individuales (Ringelmann, 1913). Se encuentra un fenómeno presente posteriormente conocido como inercia social: cuando muchas personas en grupos parecen dispuestas a dejar que pocos hagan el trabajo, originando una especie de contagio para el mínimo esfuerzo (Kravitz y Martin, 1986).

Este fenómeno también fue observado en fábricas en las que los obreros producían menos individualmente cuando eran colocados en grupos de trabajo (Marriot, 1949); las personas también se comportan de esa forma cuando se trata de responsabilidad. Cuando un individuo trabaja solo, se siente responsable por todo, cuando es colocado en un grupo de trabajo, divide esa responsabilidad (Latane y Nida, 1981). Esas suposiciones sirvieron de base para otros estudios sobre comunidades y redes sociales. 
En un estudio, la comunicación entre agricultores y vecinos se consideró frente a la posibilidad de aceptar nuevas semillas de maíz hibridas, mientras que los vendedores eran indispensables en el propósito de transmitir el conocimiento (Ryan y Neal-Grosst, 1943). Ejemplos de estudios como esos ayudaron a difundir la cuestión del contagio en redes sociales. Lo mismo ocurrió en otros estudios de la misma década, realizados con profesores, alumnos y agricultores (Alers-Montalvo, 1953) y relaciones interpersonales que ayudaron a difundir, marcar y comercializar productos (Brooks, 1957).

Es importante resaltar que aún son raros los estudios identificados que examinen el contagio de la PR en redes sociales (Scherery Cho, 2003). Es posible relacionar otros tres estudios que influenciaron la comprensión del contagio de PR en redes sociales. Así, por ejemplo, las causas y las consecuencias de la división sexual del trabajo en la agricultura se prueban usando una muestra de sociedades africanas (White, Burton y Dow, 1981); el contagio de la gripe $\mathrm{H} 1 \mathrm{~N} 1$ fue evaluado junto con el papel central de las respuestas emocionales a un riesgo percibido específico que moldeó comportamientos individuales (Prati, Pietrantoni, y Zani, 2011) y el uso de medicamentos fue evaluado según el contagio de compañeros, médicos y colaboradores (Burt, 2016). Estos estudios fueron de alguna forma influenciados por estudios económicos clásicos de contagio social (Riezler, 1944), tales como la psicología del miedo (Riezler, 1944), la psicología del pánico (Quarantelli, 1954) y el efecto Bandwagon (Leibenstein, 1950; Simon, 1954).

La cuestión del contagio y la percepción del riesgo relacionada con efectos comportamentales en la psicología económica y la economía comportamental no debe confundirse con teorías de finanzas descritas con contagio de crisis y la medida del riesgo de inversiones. En este caso específico, la cuestión sugiere la quiebra de estructuras con crisis financieras que, de forma análoga a un «efecto dominó», va repasando la crisis a los agentes involucrados (Dornbusch, Park y Claessens, 2000; Forbes y Rigobon, 2002;
Rigobon, 2002a; 2002b). El esquema conceptual para el contagio del riesgo en el consumo de viajes compartidos se presenta a continuación.

\subsection{Definición del riesgo}

La observación directa del riesgo representa un gran desafío, ya que se trata de una supervariable - del tipo de variable compleja a ser observada directamente- (Hair et al., 1998). En este estudio se enfoca en la utilización de variables manifiestas que fueron utilizadas como intervinientes. A partir de la utilización de videos, presentados por un actor, fue posible estudiar el contagio del riesgo en red. Son cuatro las variables manifiestas presentadas en videos: mantenimiento del vehículo, cancelación del viaje, educación y asedio.

- Mantenimiento del vehículo. El video duraba 1:56' y el actor relataba varios problemas de conservación del vehículo del viaje: centenas de kilómetros rodados, vehículos antiguos, aire acondicionado que no funcionaba, asientos rotos y otros ítems de seguridad. La exigencia a lo largo de los años fue reducida para incrementar la entrada de nuevos conductores (de Luca y di Pace, 2015; Minifie, 2016).

- Cancelación del viaje. El video de 2:10’ narra una situación en la que el conductor rechazaba y cancelaba un viaje, ya que no se trataba de una distancia significativa. Cuando un viaje se cancela próximo a su inicio, es posible que se produzca una pérdida relacionada con atrasos o falta a un compromiso. Eso no puede evitarse tan solo con una nota buena para el conductor en el aplicativo. Casos como este son relatados con frecuencia en países como Brasil y China (Estarque, 2017).

- Educación. La educación fue abordada en el video de 2:33'. El pasajero relata la falta de educación del conductor que se comportaba de forma inflexible. Quedó bien claro para el pasajero que el vehículo no era compartido, apenas ingresó. Casos como ese son relatados como el lado oscuro — dark side - de Uber (Jericho, 2016). 
- Asedio. En este video de 1:57’ el actor relata casos de pasajeros regresando de fiestas, en la noche, alcoholizados. En este caso son víctimas de conductores que de alguna forma asedian al pasajero. El aplicativo y las buenas notas recibidas por el conductor no sirven como garantía en estos casos (Wosskow, 2014).

A continuación, se describen las variables de medida, la configuración del experimento, los procedimientos, los materiales y la técnica de análisis de datos utilizada.

\subsection{Unidades investigadas}

Practicidad y comodidad son variables importantes en el consumo de viajes compartidos. A partir de una plataforma de servicios, conectados a smartphones, es posible estimar las tarifas, prever los horarios de llegada del vehículo, el acceso al historial de un conductor, monitorear las rutas reales y compararlas con las rutas sugeridas. Los pagos se realizan de una manera rápida y pueden verificarse en informes que contemplan rutas, tiempo de recorrido y la tarifa de cada viaje. Además de esas facilidades, el usuario tiene a su disposición un automóvil más cómodo en comparación con un viaje en ómnibus más demorado (Minifie, 2016).

Los aplicativos para economía de acceso y de compartir tienen una función que permite cancelar el pedido. Esta posibilidad produce insatisfacción del usuario, ya que puede ocurrir en cualquier momento y resultar en fallas en el plano del viaje para los involucrados en el P2P. En San Pablo, Brasil, la ciudad más poblada del hemisferio sur, es común, por ejemplo, que el conductor de Uber cancele un viaje cuando identifica el destino del pasajero. Cuando se trata de un viaje corto el conductor cancela con el objetivo de buscar otro pasajero más rentable, o sea, uno que tendrá un viaje más largo para garantizar un lucro mayor con el trayecto (Estarque, 2017).

En cuanto a la seguridad, los taxis no se perciben como medio de transporte seguro, principalmente por el público femenino. También tenemos la cuestión de los asaltos a conductores de taxis, ya que los pasajeros, por lo general, pagan con dinero en efectivo (Jericho, 2016). En este aspecto se encuentra un problema que fue decisivo para la suspensión de los servicios de Uber en Londres y otras importantes ciudades del mundo. En la capital inglesa, la seguridad ha sido la clave de las medidas contra Uber; la principal alegación ha sido que la empresa no observa potenciales implicaciones de seguridad.

La empresa Transport for London, agencia reguladora inglesa de transporte, mostró preocupación por la falta de informaciones de casos de crímenes ocurridos dentro de los carros y la forma como la empresa obtiene certificados médicos y antecedentes criminales de sus conductores (Salek, 2017). Así, algunos criterios de seguridad deberían ser seguidos, tales como pasar una ficha criminal, verificar el histórico de conducción, la necesidad de tener una concentración cero de alcohol en la sangre y carros sometidos a inspecciones apropiadas (Minifie, 2016). Aspectos como estos resultaron en la suspensión de los servicios de la empresa Uber en Londres, una ciudad con 3,5 millones de clientes y cerca de 40000 conductores que trabajan para la empresa en la capital británica (Cox, 2017; Kleinman, 2017; Topham, 2017). En el 2020 los servicios de Uber volvieron a Londres. 


\section{Metodología}

\subsection{Participantes y configuración}

El experimento fue realizado con 16 factores trade offs- en niveles de «alto» y «bajo» para las variables precio, practicidad, comodidad y seguridad. La organización general del experimento tiene tres supervariables - practicidad, comodidad y seguridad- de definición compleja (Hair et al., 1998). A fin de resolver este problema, la solución fue prever estacas para definir la complejidad de cada variable. En otras palabras, esa definición explicaría lo que es práctico, cómodo y seguro en este tipo de acceso. Las definiciones para esas variables complejas fueron repasadas a los participantes antes de aplicar el instrumento de colecta de datos. Las variables se describen a continuación.

- Practicidad. Considera aspectos tales como velocidad, uso de aplicativos, rapidez de atendimiento y acceso, entre otros.

- Comodidad. Se relaciona con el hecho de ir solo el conductor y el pasajero, poder parar en medio del camino, transporte de maletas y objetos personales, espacio interno y ausencia de pasajeros en el asiento contiguo, entre otros.

- Seguridad. Engloba violencia, uso de malas palabras y riesgos como secuestro, entre otros.

La muestra la constituyeron 30 participantes con media de uso de 12,93 meses para Carpooling y 13,92 meses para Uber. Los usuarios tenían edad media de 29,32 años y usaban esos servicios de compartir viajes para ir al trabajo - 41,38 \%-, estudios -31,03\%-y diversión - 27,59 \%-. Todos los participantes eran voluntarios y escogidos al azar, en locales de gran circulación de Goiânia-Goiás, Brasil. Al final de la explicación de lo que representaba cada supervariable — seguridad, practicidad y comodidad - se entregaba una ficha con el fin de poner una nota a cada uno de los dieciséis factores - trade offs-.

Solo participaron del estudio los que tenían experiencia frecuente de acceso de Uber, WillGo, Cabify, Televo, EasyGo y BlaBlaBlaCar, entre otros. Las fichas se entregaban a los participantes después de la exposición de cuatro videos relacionados con cuatro problemas sobre el consumo de transporte por acceso: mantenimiento del vehículo, cancelación del viaje, falta de educación y asedio.

\subsection{Modelo conceptual}

El esquema conceptual del estudio tiene como objetivo medir la relación de las variables independientes - atributos- seguridad, practicidad, comodidad, precio y la percepción del riesgo de los usuarios, a partir de cuatro variables manifiestas -riesgos-: cancelación, mantenimiento, educación y asedio. Las variables manifiestas son utilizadas en este esquema conceptual para posibilitar la observación de las variables independientes y la variable de respuesta. 
Figura 1. Modelo conceptual

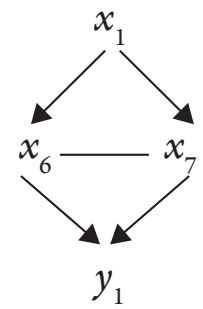

(a)

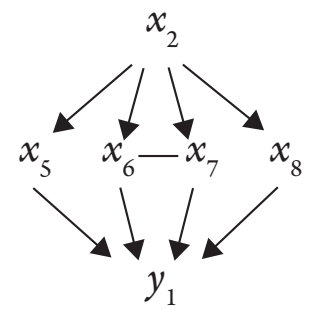

(b)

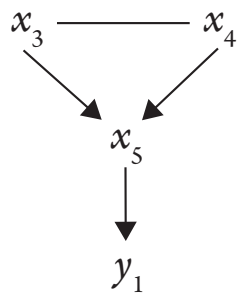

(c)

Fuente. Elaboración propia.

De acuerdo con la figura 1, existen tres tipos de variables presentes:

(a) manifestadas — riesgos-: $\mathrm{x}_{1}$-cancelación-, $\mathrm{x}_{2}-$ mantenimiento-, $\mathrm{x}_{3}-e d u c a c i o ́ n-\mathrm{e}_{4}$ -asedio-;

(b) independientes - atributos-: $\mathrm{x}_{5}$ seguridad-, $\mathrm{x}_{6}$ - practicidad-, $\mathrm{x}_{7}-$ comodidad- e $\mathrm{x}_{8}$-precio-;

(c) dependiente: $y_{1}$-percepción del riesgo-;

(d) control: edad, frecuencia de uso; finalidad de uso.

\subsection{Hipótesis de la investigación}

La hipótesis central de este estudio sugiere que la proximidad de dos actores en una comunidad de consumo se relaciona con la presencia de una influencia interpersonal entre esos actores. La teoría de contagio social indica que las percepciones y los comportamientos iniciados por un miembro de la red influenciarán a los otros en la red.

A partir de estudios relacionados se sabe que el mantenimiento, la cancelación, la educación y el asedio son los riesgos percibidos por los usuarios de Uber y viajes compartidos (de Luca y di Pace, 2015b; Minifie, 2016; Estarque, 2017; Jericho, 2016; Wosskow, 2014). Entonces, se pretende identificar cuál es la percepción de tales riesgos:
- $\mathrm{H}_{1}$ : las percepciones del riesgo para el consumo Uber son semejantes a las percepciones del riesgo del Carpooling.

- $\mathrm{H}_{2}$ : los individuos son contagiados por el riesgo cuando se ubican en la misma comunidad de consumo.

- $\mathrm{H}_{3}$ : las variables manifestadas afectan la percepción del riesgo con la misma intensidad.

\subsection{Procedimientos específicos}

Los sujetos experimentales fueron 30 individuos seleccionados por alumnos involucrados en este estudio. Los participantes actuaron como voluntarios y ninguno había participado de un estudio semejante. Para la selección de los voluntarios se entregó un cuestionario con preguntas que funcionaban como un filtro de entrada:

1. ¿Usted usa Uber en sus viajes?

2. ¿Usted utiliza viajes compartidos?

3. ¿Hace cuánto tiempo usted utiliza la opción $\mathrm{A}$ o B?

4. Sexo.

5. Edad.

6. Salario.

7. Frecuencia de uso. 
Después de realizar un sorteo, los participantes aleatorios que tenían más de diez meses de experiencia en el uso de transporte compartido eran invitados a participar del estudio. En grupos, observaban solo uno de los cuatro videos mantenimiento, cancelación, educación y asedio-. El grupo de control no fue invitado a ver los videos y apenas llenó el cuestionario.

Para el desarrollo del experimento se crearon cuatro videos, utilizando el mismo actor, con 23 años, próxima a la edad media de los participantes. En cada video el actor comentaba un problema relacionado con su experiencia de consumo. En el video 1 -mantenimiento-, de 1:56 minutos, el vehículo tenía neumáticos con desgaste excesivo; algunas señales mostraban muchos años de uso aire acondicionado, ventanas eléctricas y puertas con defecto-. En el video 2 - cancelación-, de 2:10 minutos, el actor presentaba una situación en la que ocurrían cancelaciones de viajes por parte del prestador del servicio. Situaciones como perder compromisos, por lo general, son resultado de esa práctica. En el video 3 -educación-, de 2:03 minutos, se presentaba una situación que involucraba a un conductor que fumaba y utilizaba lenguaje obsceno. En el video 4 -asedio-, de 1:57 minutos, prevalecía una situación de asedio, en la que el conductor hacía insinuaciones y confrontaba al pasajero.

Para presentar los cuatro videos se utilizó un computador portátil, parlantes y un proyector, instalados en un pequeño auditorio. Los participantes eran separados durante las presentaciones con el fin de evitar el intercambio de información con las personas que no estaban involucradas en ese momento. Después de cada presentación, un cuestionario se entregaba a fin de que lo diligenciaran. El instrumento de colecta de datos tenía 16 líneas que presentaban combinaciones entre los niveles alto y bajo para cada una de las variables independientes atributos-: seguridad, practicidad, comodidad y precio. La estructura fue orientada de la siguiente manera:
- seguridad: alta y baja;

- practicidad: alta y baja;

- comodidad: alto y bajo;

- precio: alto y bajo.

El uso de las variables manifiestas - previstas en los cuatro videos, mantenimiento, cancelación, educación y asedio - permitió medir las variables independientes seguridad, practicidad, comodidad y precio.

La selección de los estímulos se realizó a partir de la selección entre 16 niveles - low, L y high, $\mathrm{H}, 2^{*} 2^{*} 2^{*} 2$ - En ese proceso dos trade offs considerados impracticables fueron excluidos. El participante atribuía una nota de 1 a 9 para cada perfil del análisis coyuntural. La selección se realizó después de que los participantes observaron los videos seleccionados. Un grupo de entrevistados no vio los videos y ese grupo representó al grupo de control (GC).

El análisis de los datos se llevó a cabo mediante el empleo del conjoint análysis. Esa técnica multivariada resulta adecuada en el análisis de los atributos en situación de trade off para fines de calidad, desarrollo de productos, segmentación de mercado o estudio de marcas (Green y Srinivasan, 1990; 1978; Johnson y Olberts, 1996; Wittink y Cattin, 1989).

El primer paso en el análisis fue el estudio de los valores de parte (parth worths) de los cinco grupos - control, cancelación, asedio, educación y mantenimiento- Después, la importancia relativa del factor - relative importance offactor - fue refinada. La tercera etapa confrontó a los cuatro grupos experimentales con el grupo de control. Con el fin de verificar la precisión predictiva del modelo se utilizó el parámetro Rô de Spearman —estimación-y el Tau de Kendal —validación-.

Los sujetos fueron separados en grupos y sometidos a niveles distintos de las variables independientes, utilizando un efecto de diseño del sujeto único. Los cuatros videos fueron presentados grupalmente, sin 
embargo, los cuestionarios se aplicaron de manera individual. La preocupación se debe al hecho de que eventuales efectos de comportamiento de grupo podrían influenciar las respuestas. Sería posible, por ejemplo, que durante ese periodo de estudio alguna noticia sobre violencia en viajes compartidos fuera divulgada como noticia, se trata de una forma de agregar más control. Además de las variables de control empleadas que se han citado —edad, frecuencia de uso y finalidad de uso-. El uso de videos cortos, que variaban de 1:56 a 2:10 minutos, evitó el efecto fatiga en las respuestas. El mismo procedimiento fue adoptado con los cuestionarios que tenían una cantidad reducida de 16 trade offs.

\section{Análisis}

La primera etapa de análisis de datos continuó identificando si existe diferencia entre las medias para los grupos Carpooling y Uber (Tabla 1). De esta forma, se atiende a las exigencias de $\mathrm{H} 1$-las percepciones del riesgo para el consumo Uber son semejantes a las percepciones de riesgo del Carpooling-.

Tabla 1. Comparación de medias

\begin{tabular}{|c|c|c|c|c|c|c|c|c|}
\hline & \multicolumn{4}{|c|}{ Uber } & \multicolumn{4}{c|}{ Carpooling } \\
\hline & Precio & Practicidad & Comodidad & Seguridad & Precio & Practicidad & Comodidad & Seguridad \\
\hline Media & 4,55 & 4,99 & 3,81 & 3,45 & 4,29 & 4,55 & 4,30 & 4,55 \\
\hline Variancia & 3,27 & 3,27 & 3,09 & 3,83 & 0,49 & 2,66 & 2,49 & 3,90 \\
\hline $\begin{array}{c}\text { Desviación } \\
\text { Estándar }\end{array}$ & 1,81 & 1,81 & 1,76 & 1,96 & 0,70 & 1,63 & 1,58 & 1,97 \\
\hline Error & 0,87 & 0,87 & 0,82 & 1,02 & 0,13 & 0,71 & 0,66 & 1,04 \\
\hline
\end{tabular}

Fuente. Elaboración propia.

De acuerdo con la tabla 1, los riesgos percibidos para ambas muestras evidenciaron una diferencia no muy significativa con respecto a las dispersiones en torno a las medias para los 14 trade offs - p: 0,21; F: 1,41; $\mathrm{F}_{\text {crítico }}: 2,01-$. A partir de esa comprensión, fue evidente que los consumidores de Carpooling y de Uber adoptan un proceso de selección semejante cuando son expuestos a los riesgos percibidos de mantenimiento, asedio, cancelación y educación. El grupo de control - ${ }^{*}$ - presenta una desviación estándar considerado bajo $-0,91$ - en torno a la media $-4,77$ - 
Figura 2. Desviación estándar

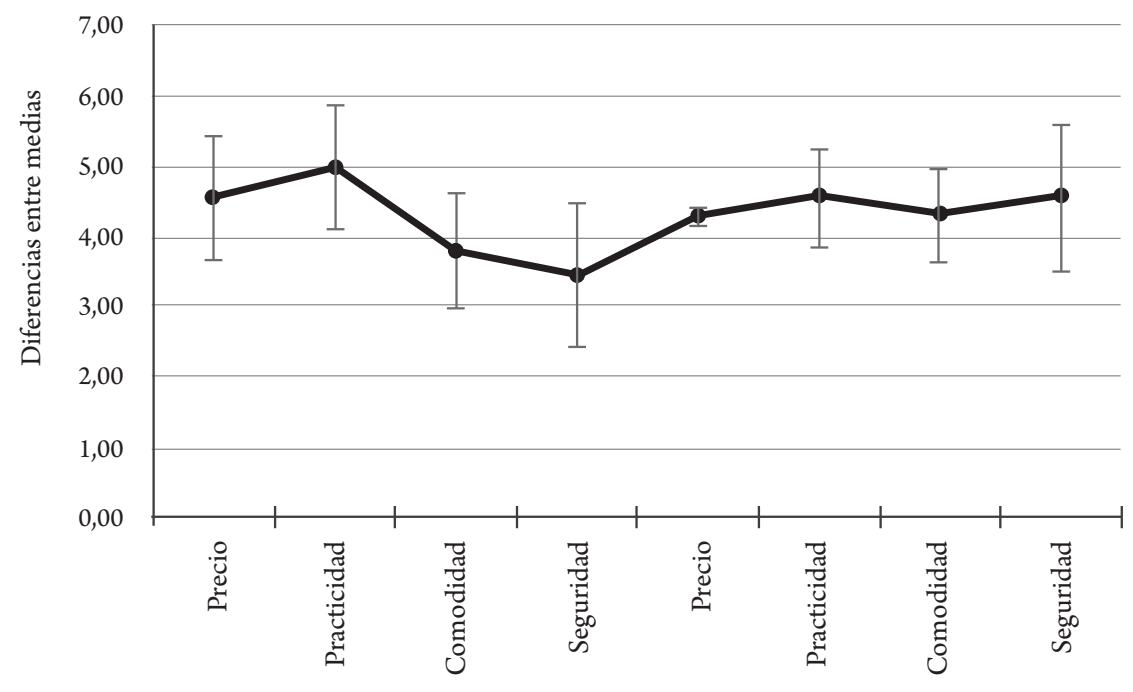

Fuente. Elaboración propia.

La segunda etapa del estudio analizó los catorce trade offs para percepción del riesgo a partir del análisis coyuntural, identificando las utilidades parciales, las utilidades totales y la precisión predictiva del modelo. Los análisis fueron realizados para los grupos Carpooling y Uber.

En la tabla 2 se presentan los cuatro factores y sus ocho niveles que, juntos, representan el estímulo para la percepción del riesgo. El objetivo de esta etapa es observar si los participantes son sensibles a la manipulación de las probabilidades de refuerzo a través de la verificación de la distribución de las respuestas. En otras palabras, es posible entender cómo ocurre la distribución de cada PR a medida que los individuos son contagiados con uno de los cuatro riesgos posibles.

Tabla 2. Factores y niveles

\begin{tabular}{|c|c|c|c|c|c|c|c|c|c|c|c|}
\hline & \multicolumn{5}{|c|}{ Carpooling } & \multicolumn{5}{|c|}{ Uber } \\
\hline & & Pre & Pract & Com & Seg & $\mathrm{R}$ & Pre & Pract & Com & Seg & $\mathrm{R}$ \\
\hline \multirow{2}{*}{ Mantenimiento } & (1) & 0,27 & 0,21 & 0,54 & 1,02 & \multirow{2}{*}{0,697} & 0,196 & 0,577 & 0,83 & 0,89 & \multirow{2}{*}{0,650} \\
\hline & (2) & 13,46 & 10,47 & 26,28 & 49,78 & & 7,86 & 23,14 & 33,24 & 35,77 & \\
\hline \multirow{2}{*}{ Asedio } & (1) & 0,08 & 0,40 & 0,49 & 1,19 & \multirow{2}{*}{0,737} & 0,26 & 0,01 & 0,14 & 1,37 & \multirow{2}{*}{0,891} \\
\hline & (2) & 4,00 & 18,7 & 22,54 & 54,69 & & 14,69 & 0,06 & 7,63 & 77,63 & \\
\hline \multirow{2}{*}{ Cancelación } & (1) & 0,45 & 0,71 & 0,01 & 1,07 & \multirow{2}{*}{0,651} & 0,402 & 0,17 & 0,23 & 0,388 & \multirow{2}{*}{0,606} \\
\hline & $(2)$ & 20,35 & 31,75 & 0,27 & 47,63 & & 33,87 & 14,07 & 19,38 & 32,69 & \\
\hline \multirow{2}{*}{ Educación } & (1) & 0,14 & 0,08 & 0,135 & 0,50 & \multirow{2}{*}{0,746} & 0,043 & 0,017 & 0,22 & 0,022 & \multirow{2}{*}{0,874} \\
\hline & (2) & 16,88 & 9,48 & 15,61 & 58,03 & & 14,73 & 2,40 & 75,34 & 7,530 & \\
\hline
\end{tabular}

(1) Los valores son referentes al valor de parte — parth worth—, mayores valores expresan más utilidad en su nivel de atributo. (2) Los valores son expresados en porcentuales, indicando la cantidad total de utilidad distribuida entre cada atributo.

Pre: Precio - Pract: Practicidad - Com: Comodidad - Seg: Seguridad

Fuente. Elaboración propia. 
- Riesgo percibido mantenimiento. Cuando el video «mantenimiento» es mostrado al grupo Carpooling, las preferencias centradas en seguridad son evidentes - $49,78 \%$ - Ocurre lo mismo en el grupo Uber - $35,77 \%$ - sin embargo, en ese agrupamiento la concentración de utilidad total se concentra también para comodidad - 33,24 \%-.

- Riesgo percibido asedio. Cuando el video «asedio» fue mostrado al grupo Carpooling, ocurre una situación semejante con la selección concentrada en el atributo seguridad $-54,69 \%$ - La seguridad también representa la principal elección para el grupo Uber $-77,63 \%$ -

- Riesgo percibido cancelación. Cuando el vídeo «cancelación» se muestra al grupo Carpooling, la elección se concentra en el atributo seguridad -47,63\%—, sin embargo, en ese grupo, la concentración de utilidad total se concentra también para practicidad -31,75\%—. En el grupo Uber el atributo «precio» - 32,69\%divide la elección con el atributo «seguridad» $-32,69 \%$ -

- Riesgo percibido educación. Cuando el video «educación» se muestra al grupo Carpooling, la elección se concentra en el atributo seguridad - 58,03 \%—. La «seguridad» también representa la principal elección para el grupo Uber - 75,34 \%-.

Se entiende que el modelo ampliado de contagio de riesgo, para ambos grupos, tiene la seguridad como principal atributo. Sin embargo, algunas anotaciones deben hacerse sobre las utilidades parciales. Cuando los valores de parte - parth worths - son ordenados en orden creciente, se tiene un orden de importancia (Hair et al., 1998).

De acuerdo con ese criterio, la «seguridad» es el aspecto más importante en todos los casos para Uber y Carpooling, excepto para la situación de «asedio» en el grupo Uber y «educación» — falta de- en el grupo Carpooling.

Tabla 3. Valores de Parte — parths worths-

\begin{tabular}{|c|c|c|c|c|}
\hline & \multicolumn{2}{|c|}{ Carpooling } & \multicolumn{2}{c|}{ Uber } \\
\hline Variable manifiesta & Variable independiente & Valor de parte & Variable independiente & Valor de parte \\
\hline Mantenimiento & Seguridad & $\underline{1,017}$ & Seguridad & 1,074 \\
\hline Mantenimiento & Comodidad & 0,537 & Practicidad & 0,716 \\
\hline Mantenimiento & Precio & 0,275 & Precio & 0,459 \\
\hline Mantenimiento & Practicidad & 0,214 & Comodidad & 0,006 \\
\hline Asedio & Seguridad & $\underline{0,892}$ & Precio & $\underline{0,402}$ \\
\hline Asedio & Comodidad & 0,829 & Seguridad & 0,388 \\
\hline Asedio & Practicidad & 0,577 & Comodidad & 0,230 \\
\hline Asedio & Precio & 0,196 & Practicidad & 0,167 \\
\hline Cancelación & Seguridad & $\underline{1,196}$ & Seguridad & $\underline{0,502}$ \\
\hline Cancelación & Comodidad & 0,493 & Precio & 0,146 \\
\hline Cancelación & Practicidad & 0,409 & Comodidad & 0,135 \\
\hline Cancelación & Precio & 0,089 & Practicidad & 0,082 \\
\hline Educación & Seguridad & $\underline{1,374}$ & Comodidad & $\underline{0,220}$ \\
\hline Educación & Precio & 0,260 & Precio & 0,043 \\
\hline Educación & Comodidad & 0,135 & Seguridad & 0,022 \\
\hline Educación & Practicidad & 0,001 & Practicidad & 0,007 \\
\hline
\end{tabular}

Fuente. Elaboración propia. 
Tenemos que en el grupo Uber las utilidades parciales más importantes están relacionadas con seguridad, a partir de la tabla 2. Para el usuario de Uber, las situaciones de educación — falta de- y cancelación provocan las mayores situaciones de inseguridad. Se destaca que, entre las variables manifestadas, el asedio es el factor de menor utilidad parcial para el aspecto «seguridad».

El análisis relacionado con el grupo Carpooling tiene utilidades parciales para seguridad más bajas en contraste con el grupo Uber, a partir de la tabla 2. En otras palabras, los participantes del grupo Carpooling se sienten más seguros que los participantes del grupo Uber. Para ese grupo, existen dos diferencias significativas que alteran el cuadro de contagio de riesgo tradicional, centrado en la seguridad. Cuando ese usuario se encuentra en situación de asedio, percibe el riesgo en precio, en vez de seguridad conforme al grupo Carpooling. En situación de educación - falta de- ese participante percibe el riesgo en comodidad, en vez de seguridad conforme al grupo Carpooling.
La tabla 2 también posibilita un análisis de valor para los niveles de las variables relacionadas (Hair et al., 1998). Al ordenar todas las utilidades parciales, en los grupos Uber y Carpooling, podemos tener un escenario de importancia general para el estudio. Por tanto, las variables más importantes en sus niveles son: 1) educación-seguridad -1,374-Carpooling —; 2) cancelación-seguridad -1,196-Carpooling-; 3) mantenimiento-seguridad -1,074-Uber-; 4) mantenimiento-seguridad -1,017-Carpooling-; 5) asedio-seguridad -0,892-Carpooling-; 6) asedio-comodidad -0,829-Carpooling-; 7) mantenimiento-practicidad -0,716-Uber-; 8) asedio-practicidad -0,577-Carpooling - ; 9) mantenimiento-comodidad -0,537-Carpooling_-; 10) cancelación-seguridad $-0,502-$-Uber-.

Al ordenar las utilidades parciales podemos ver a los participantes del grupo Carpooling como los que atribuyen más valor para la $\mathrm{PR}$ de seguridad, principalmente, en el aspecto de educación, cancelación y mantenimiento.

Figura 3. Variable mantenimiento

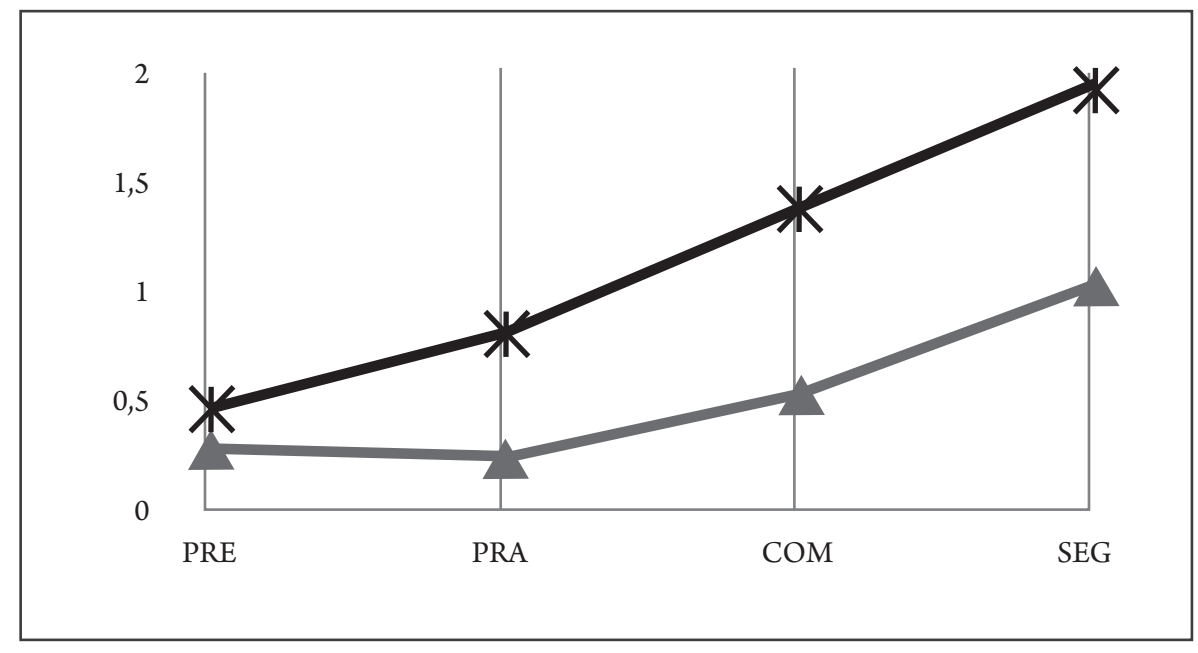

Nota. X - Uber

* - Carpooling

Fuente. Elaboración propia. 
Figura 4. Variable asedio

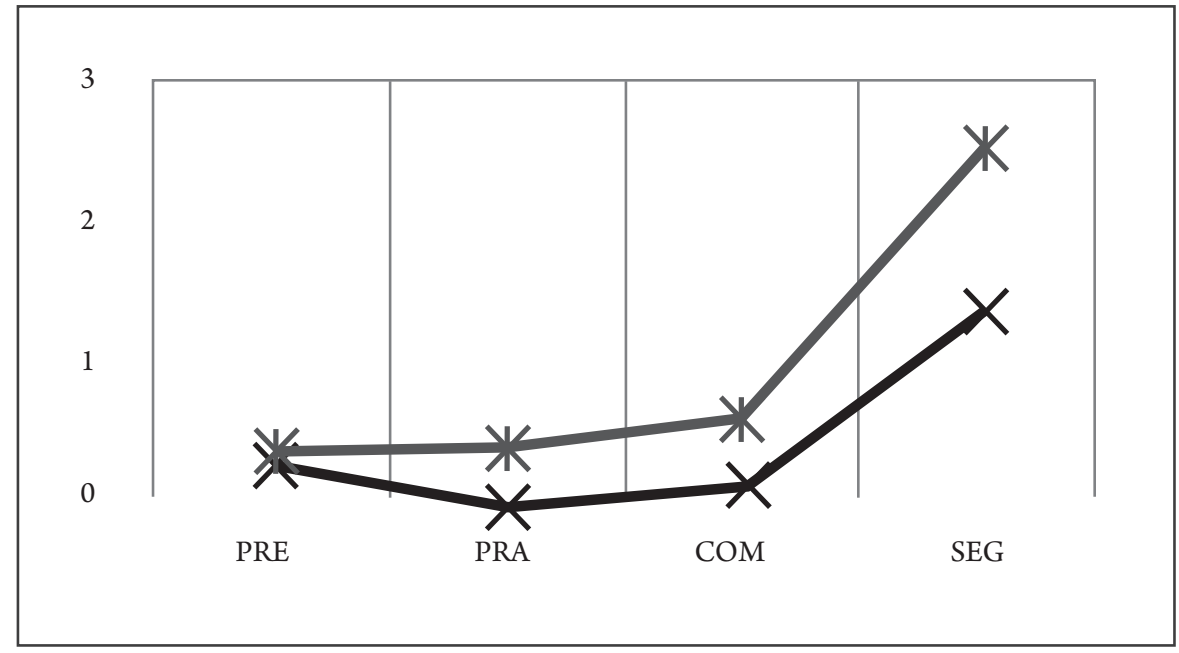

Nota. X - Uber

* - Carpooling.

Fuente. Elaboración propia.

Figura 5. Variable cancelación

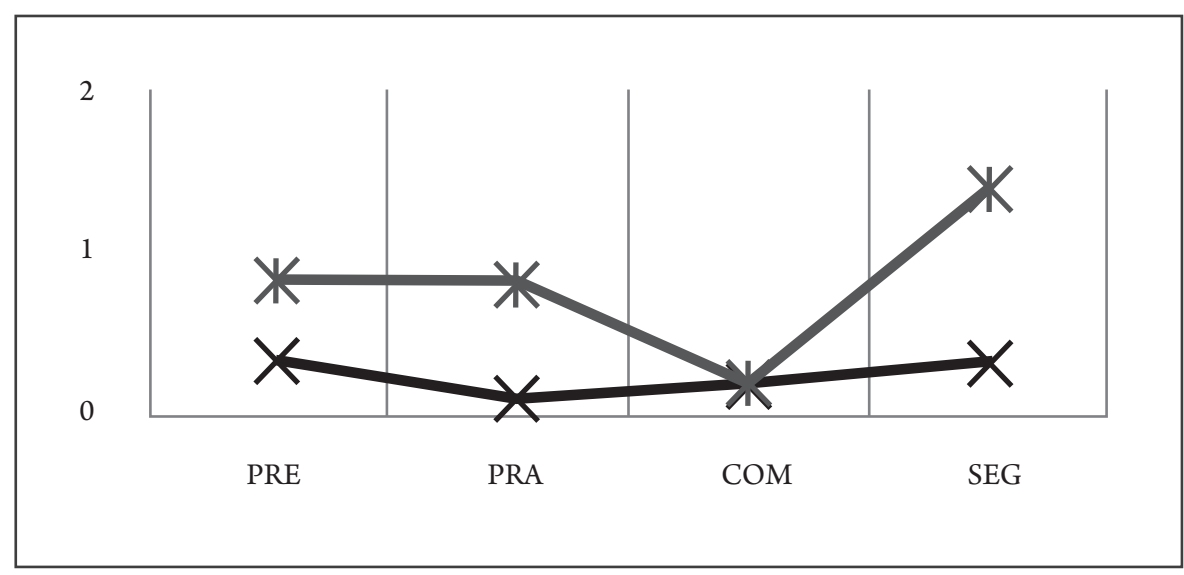

Nota. X - Uber

$$
\text { * - Carpooling. }
$$

Fuente. Elaboración propia. 
Figura 6. Variable educación

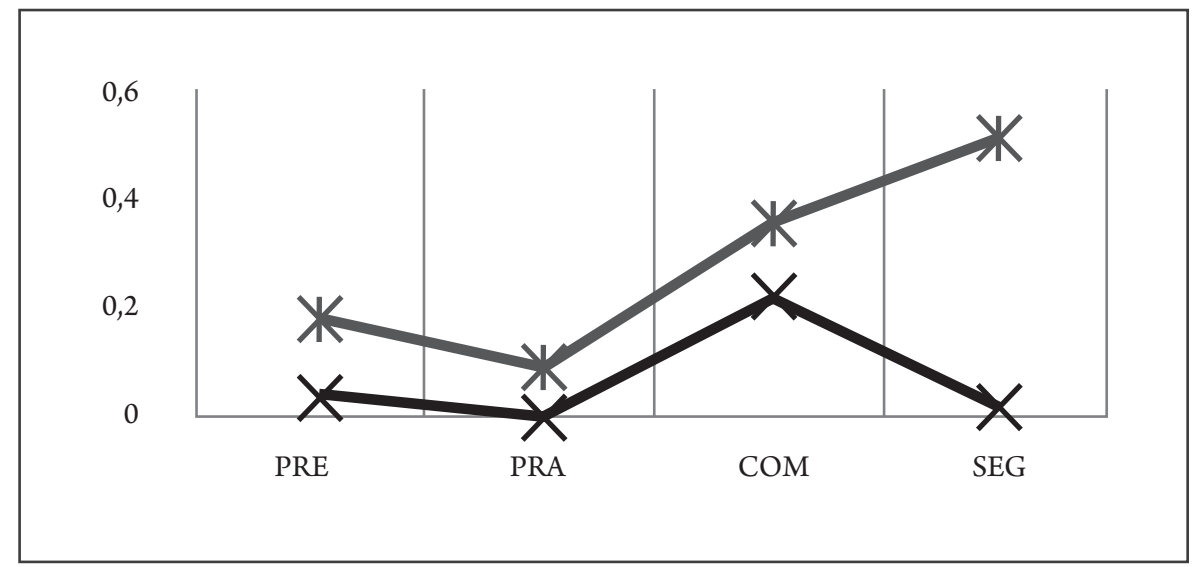

Nota. X - Uber

* - Carpooling.

Fuente. Elaboración propia.

Las figuras 3, 4, 5 y 6 contemplan las utilidades parciales para los cuatro riesgos analizados. El contagio del riesgo percibido «mantenimiento» (véase la Figura 2) muestra un valor semejante para «precio» en los grupos Carpooling y Uber, pero ese comportamiento se va alterando a medida que «seguridad» asume la posición de principal riesgo percibido. La $\mathrm{PR}$ «seguridad» es más importante para el grupo Carpooling. Con relación al grupo Uber, cuando se percibe que un vehículo tiene problemas de mantenimiento, los atributos «precio»y «practicidad» registran utilidades próximas a cero.

El contagio del riesgo percibido «asedio» (véase la Figura 3) indica también un valor semejante para «precio» en los grupos Carpooling y Uber, pero ese comportamiento se va alterando a medida que «seguridad» asume la posición de principal PR. El riesgo percibido «seguridad» es más importante para el grupo Carpooling. Cabe destacar que para el riesgo percibido «asedio» tenemos el mayor valor para el atributo «seguridad» entre todos los riesgos percibidos. Con relación al grupo Carpooling, cuando se percibe la situación de «asedio», los atributos «precio», «practicidad» $y$ «comodidad» registran utilidades próximas a cero.
El contagio de la PR «cancelación» (véase la Figura 4) indica un valor semejante para «comodidad» en los grupos Carpooling y Uber. La percepción de «precio»y «diversión» registran utilidades parciales más bajas para el grupo Uber. La $\mathrm{PR} \ll$ seguridad» es más importante para el grupo Carpooling. En el contagio de la PR «cancelación» están las mayores dispersiones de utilidad parcial de todas las percepciones de la PR. En otras palabras, expuestos al riesgo de cancelación de un viaje, los usuarios de Carpolling y Uber perciben los atributos «precio», «practicidad»y «seguridad» de formas distintas.

El contagio del riesgo percibido «educación» (véase la Figura 5) indica un valor semejante para «practicidad» en los grupos Carpooling y Uber. La percepción de «precio», «practicidad»y «comodidad» es semejante para los grupos Uber y Carpooling. El riesgo percibido «seguridad» es más importante para el grupo Carpooling, en este riesgo percibido se encuentra la mayor diferencia para la percepción de «seguridad» entre las cuatro PR analizadas, o sea, en la presencia de la falta de educación, el consumidor de viajes compartidos se siente más inseguro que el consumidor de Uber. 
Con relación al grupo Uber, cuando se percibe la situación «educación» — falta de—, los atributos «precio», «practicidad»y «seguridad» registran utilidades próximas a cero.

El modelo de análisis coyuntural presenta un ajuste adecuado — poder explicativo de la regresión-, con valores de determinación variando entre 0,606 a 0,891.

\section{Discusión de los resultados}

$\mathrm{E}^{\mathrm{I}}$ consumo de viajes compartidos se desarrolló con el fin de agilizar y reducir costos en la vida de los viajeros, además de disminuir los embotellamientos, la contaminación, las emisiones y la demanda por infraestructura de estacionamiento (Fellows y Pitfield, 2000; Stiglic et al., 2016). Compartir viajes también puede desempeñar un papel importante en la redistribución del alquiler proporcionando a la población pobre la oportunidad de acceso a servicios de transporte baratos o empleos secundarios (Li, Taeihagh y De Jong, 2018). Algunas externalidades surgieron junto con esas oportunidades y algunos riesgos percibidos aparecieron.

En este estudio la seguridad se considera el riesgo percibido más importante. El segundo atributo más importante es la comodidad. Cuando un viaje se cancela o hay falta de educación en el servicio Uber, el usuario no se siente inseguro, pero sí en una situación incómoda. La falta de mantenimiento de los vehículos genera contagio en la percepción del riesgo percibido para comodidad y seguridad, principalmente en el grupo Carpooling. Cabe resaltar la importancia con relación a los riesgos percibidos para asedio $-77,63 \%$ de utilidad total en el grupo Uber- y falta de educación - 75,34 $\%$ de utilidad total en el grupo Uber-. En todas las situaciones de contagio de riesgo percibido el precio pierde su importancia como atributo central, favoreciendo una situación de inseguridad o incomodidad.

Algunos aspectos sugieren estudios futuros más profundos. Cuando se está en situación de contagio del riesgo de cancelación, el usuario no se muestra en situación de incomodidad, pero sí inseguro. Eso sugiere inseguridad con relación a compromisos asumidos — trabajo o diversión-. Los resultados del contagio de la PR mantenimiento apuntan algunas ideas. Comodidad —falta de- en equilibrio con seguridad - falta de- sugiere que, en el caso de falta de mantenimiento de un vehículo, surja incomodidad como atributo clave en vez de percepciones de riesgos de accidentes con el vehículo. Los puntos evidenciados en este estudio también indican mejoras en las prácticas de consumo de viajes compartidos.

El sistema de viajes compartidos fue desarrollado para ser práctico y barato, $\mathrm{y}$ algunos aspectos relacionados con seguridad y comodidad fueron desconsiderados. Hay riesgos percibidos presentes que podrían ser minimizados, para conductores y pasajeros, cuando un viaje se inicia. De un lado, el registro y la selección de conductores podría ser más riguroso y el sistema de notas podría ser más específico. En Los Ángeles, los conductores de taxi deben tener las huellas digitales registradas y deben pasar por una verificación nacional de antecedentes criminales del FBI.

La revisión para verificar el estado de mantenimiento del vehículo se realiza en el inicio del contrato de conductor, cuando debería ser una rutina. El riesgo percibido también es de orden financiero, ya que, difícilmente, la empresa Uber se presenta como responsable cuando algo sale mal en los viajes (Bawden-Davis, 2019). Algunos criterios de seguridad que se siguen también en Londres, para conductores de taxi, podrían ser extendidos al caso de los viajes compartidos: pasar un histórico criminal del conductor, verificar el histórico de 
conducción, tener concentración cero de alcohol en la sangre e inspecciones apropiadas para los vehículos (Minifie, 2016).

Sobre el sistema de notas que evalúa usuarios y conductores, en sistemas de viajes compartidos como BlaBlaBla Car, SmartMumbaikar y Tripda aun la validación del perfil se realiza a partir de redes sociales como Facebook. Tales sistemas no se responsabilizan por informaciones falsas, incompletas, imprecisas, engañosas o fraudulentas, de modo que se deja a cargo de sus usuarios todo el riesgo percibido y asumido de un viaje (Chadha, 2015). Ese sistema de validación hace de los viajes compartidos objeto de crímenes e infracciones.

\section{Conclusiones}

$\mathrm{E}$ n esta investigación se estudió la percepción de riesgo en el consumo de viajes compartidos. El mayor impacto de los hallazgos está relacionado con mudanzas en el mercado de viajes ofrecidos por los taxis. La ubereconomía ya alteró varios sectores tradicionales de la industria de bienes y servicios.

Los resultados de este estudio sugieren nuevas direcciones para estudios futuros. Se considera la seguridad y la comodidad como principales aspectos, por tanto, algunas externalidades podrían ser trabajadas. Las personas deben salir más de casa, en función de la comodidad y el costo bajo para ese tipo de viaje; por lo tanto la tasa de robo de vehículos podría verse afectada, ya que los vehículos estarían en los garajes o en locales más seguros —habrá más lugares disponibles en las calles - Los accidentes de tránsito fatales tienden a disminuir, al final los conductores de Uber y los de la competencia poseen una conducción técnica y experimentada (Dills y Mulholland, 2018).

El consumo de alcohol por parte de los usuarios podría incrementar, debido a la seguridad que el viaje compartido proporciona. En cuanto al consumo, se ha podido establecer que más crímenes están relacionados con el consumo de drogas lícitas; por lo que si las personas pasarán menos tiempo en las paradas de ómnibus, esperando el horario de embarque, podría concluirse que a menor interacción social, menor criminalidad (Skinner y Staiger, 2015).

Dado que la seguridad y la comodidad son atributos clave, cabe desarrollar funciones en las plataformas de acceso que tengan como objetivo mejorar los aplicativos, principalmente, en la de evaluación de los involucrados y en los indicadores de mantenimiento de los vehículos. La mejoría de los sistemas puede ser extendida al riesgo percibido «cancelación», el cual tiene influencia en la percepción del precio y torna las relaciones inseguras. Así ocurrió en Londres cuando el aplicativo fue prohibido, pero en el 2020 volvió con varias funcionalidades revisadas y perfeccionadas para seguridad de los involucrados.

El sistema de notas que sirve para la evaluación de los involucrados en el sistema de viajes compartidos puede distinguir cuestiones de riesgo percibido para «asedio»y «educación» - falta de-, ya que tales riesgos percibidos en contagio reciben importancias distintas, pues el asedio torna un viaje inseguro, mientras que la falta de educación torna un viaje incómodo.

Así las cosas, se puede establecer que estas lecciones deben servir para todo y cualquier servicio disponible en la economía compartida, dado que involucra los atributos de cualquier cambio P2P. 


\section{Referencias}

Alers-Montalvo, M. (1953). Cultural Change in a Costa Rican Village. Human Organization, 15(4), 2-7. DOI: https://doi. org/10.17730/humo.15.4.u15187u714120277

Bardhi, F.; Eckhardt, G. M. (2012). Access-based consumption: the case of car sharing. Journal of Consumer Research, 39(4), 881-898. DOI: https://doi.org/10.1086/666376

Bawden-Davis, J. (27 de agosto de 2019). 5 reasons why Uber can be a risky choice (for drivers and passengers). Supermoney. Recuperado de https://bit.ly/3erbGsY

Brooks, R. C. (1957). «Word-of-mouth» advertising in selling new products. Journal of Marketing, 22(2), 154-161. DOI: https://doi.org/10.2307/1247212

Burt, R. S. (2016). Social contagion and innovation: cohesion versus structural equivalence. American Journal of Sociology, 92(6), 1287-1335. DOI: https://doi.org/10.1086/228667

Chadha, S. (15 de enero de 2015). Use entirely at your own risk: 4 reasons why I will not use car-pooling service BlaBlacars. Firstpost. Recuperado de https://bit.ly/3ttspQP

Cox, J. (13 de octubre de 2017). Uber ban: firm to continue operating in London after filing appeal. Independent. Recuperado de https://bit.ly/3ttiYkg

Cox, L. A. (2012). Confronting deep uncertainties in risk analysis. Risk Analysis, 32(10), 1607-1629. DOI: https://doi. org/10.1111/j.1539-6924.2012.01792.x

de Luca, S.; di Pace, R. (2015). Modelling users' behaviour in inter-urban carsharing program: a stated preference approach. Transportation Research Part A: Policy and Practice, 71, 59-76. DOI: https://doi.org/10.1016/j.tra.2014.11.001

Dills, A. K.; Mulholland, S. E. (2018). Ride-sharing, fatal crashes, and crime. Southern Economic Journal, 84(4), 965-991. DOI: https://doi.org/10.1002/soej.12255

Dornbusch, R.; Park, Y. C.; Claessens, S. (2000). Contagion: understanding how it spreads. The World Bank Research Observer, 15(2), 177-197. DOI: https://doi.org/10.1093/ wbro/15.2.177

Douglas, M.; Wildavsky, A. (1982). How can we know the risks we face? Why risk selection is a social process. Risk Analysis, 2(2), 49-58. DOI: https://doi.org/10.1111/j.1539-6924.1982. tb01365.x

Estarque, M. (18 de septiembre de 2017). Cancelamento de corrida vira «febre» em Cumbica e irrita clientes da Uber. Folha de São Paulo. Recuperado de https://bit.ly/2R0Wqdq

Fellows, N. T.; Pitfield, D. E. (2000). An economic and operational evaluation of urban car-sharing. Transportation Research Part D: Transport and Environment, 5(1), 1-10. DOI: https://doi. org/10.1016/S1361-9209(99)00016-4
Forbes, K.; Rigobon, R. (2002). No contagion, only interdependence: measuring stock market comovements. American Finance Association, 57(5), 2223-2261. DOI: https://doi.org/10.1111/0022-1082.00494

Green, P. E.; Srinivasan, V. (1978). Conjoint analysis in consumer research: issues and outlook. Journal of Consumer Research, 5(2), 103-123. DOI: https://doi.org/10.1086/208721

Green, P. E.; Srinivasan, V. (1990). Conjoint analysis in marketing: new developments with implications for research and practice. Journal of Marketing, 54(4), 3-19. DOI: https:// doi.org/10.2307/1251756

Guidotti, R.; Nanni, M.; Rinzivillo, S.; Pedreschi, D.; Giannotti, F. (2017). Never drive alone: boosting carpooling with network analysis. Information Systems, 64, 237-257. DOI: https://doi. org/10.1016/j.is.2016.03.006

Hair, J. F.; Black, W.; Babin, B. J.; Anderson, R. E. (1998). Multivariate data analysis (5a ed.). Nueva York: Prentice Hall.

Jericho, G. (18 de abril de 2016). The dark side of Uber: why the sharing economy needs tougher rules. The Guardian. Recuperado de https://bit.ly/3h9dHMa

Joffe, H. (2003). Risk: from perception to social representation. The British Journal of Social Psychology, 42(1), 55-73. DOI: https://doi.org/10.1348/014466603763276126

Johnson, R. M.; Olberts, K. A. (1996). Using conjoint analysis in pricing studies: is one price variable enough? Research Paper Series. Sequim: Sawtooth Software. Recuperado de https://bit. ly/3bdJcRI

Kasperson, R.E.; Renn, O.; Slovic, P.; Brown, H. S.; Emel,J.; Goble, R.; Kasperson, J.; Ratick, S. (1988). The social amplification of risk-a conceptual framework. Society for Risk Analysis, 8(2), 177-187. DOI: https://doi.org/10.1111/j.1539-6924.1988. tb01168.x

Kleinman, M. (20 de octubre de 2017). Uber hails ex-Ofcom chief to help drive London licence appeal. SkyNews. Recuperado de https://bit.ly/3f4nP6s

Kravitz, D. A.; Martin, B. (1986). Ringelmann rediscovered. The original article. Journal of Personality and Social Psychology, 50(5), 936-941. DOI: https://doi.org/10.1037/00223514.50.5.936

Latane, B.; Nida, S. (1981). Ten years of research on group size and helping. Psychological Bulletin, 89(2), 308-324. DOI: https://doi.org/10.1037/0033-2909.89.2.308

Leibenstein, H. (1950). Bandwagon, Snob, and Veblen effects in the theory of consumers' demand. The Quarterly Journal of Economics, 64(2), 183-207. DOI: https://doi. org/10.2307/1882692 
Li, Y.; Taeihagh, A.; De Jong, M. (2018). The governance of risks in ridesharing: a revelatory case from Singapore. Energies, 11(5), 1277. DOI: https://doi.org/10.3390/en11051277

Marcoux, J-S. (2009). Escaping the gift economy. Journal of Consumer Research, 36(4), 671-685. DOI: https://doi. org/10.1086/600485

Marriot, R. (1949). Size of working group and output. Occupational Psychology, 23(1), 47-57.

Masuda, J. R.; Garvin, T. (2006). Place, culture, and the social amplification of risk. Risk Analysis, 26(2), 437-454. DOI: https://doi.org/10.1111/j.1539-6924.2006.00749.x

Minifie, J. (2016). Peer-to-peer pressure policy for the sharing economy. Grattan Institute. Recuperado de https://bit. ly/3w3rkB5

Murphy, D. M.; Paté-Cornell, E. (1996). The SAM framework: modeling the effects of management factors on human behavior in risk analysis. Risk Analysis, 16(4), 501-515. https://doi.org/10.1111/j.1539-6924.1996.tb01096.x

Paté-Cornell, E. (2012). On «black swans» and «perfect storms $\gg$ : risk analysis and management when statistics are not enough. Risk Analysis, 32(11), 1823-1833. DOI: https://doi. org/10.1111/j.1539-6924.2011.01787.x

Prati, G.; Pietrantoni, L.; Zani, B. (2011). A social-cognitive model of pandemic influenza H1N1 risk perception and recommended behaviors in Italy. Risk Analysis, 31 (4), 645-656. DOI: https://doi.org/10.1111/j.1539-6924.2010.01529.x

Quarantelli, E. L. (1954). The nature and conditions of panic. American Journal of Sociology, 60(3), 267-275. DOI: https:// doi.org/10.1086/221536

Riezler, K. (1944). The social psychology of fear. American Journal of Sociology, 49(6), 489-498. DOI: https://doi. org/10.1086/219471

Rigobon, R. (2002a). Contagion: how to measure it? En S. Edwards; J. A. Frankel (Eds.) Preventing currency crises in emerging markets. (269-234). Chicago: University of Chicago Press. Recuperado de https://bit.ly/3bbRpps

Rigobon, R. (2002b). The curse of non-investment grade countries. Journal of Development Economics, 69(2), 423-449. DOI: https://doi.org/10.1016/S0304-3878(02)00095-0

Ringelmann, M. (1913). Recherches sur les moteurs animés: Travail de l'homme. Annales de l'Institut National Agronomique, 12(2), 1-40. Recuperado de https://bit.ly/3uwyyge

Ryan, B.; Neal Grosst. (1943). The diffusion of hybrid seed corn in two Iowa communities. Rural Sociology, 8(1), 15-24.

Salek, S. (22 de septiembre de 2017). Por que o Uber não vai mais poder operar em Londres. BBC. Recuperado de https://bbc. in/3bamo5f

Scherer, C. W.; Cho, H. (2003). A social network contagion theory of risk perception. Risk Analysis, 23(2), 261-267. DOI: https://doi.org/10.1111/1539-6924.00306
Simon, H. A. (1954). Bandwagon and underdog effects and the possibility of election predictions. The Public Opinion Quarterly, 18(3), 245-253. DOI: https://doi.org/10.1086/266513

Skinner, J.; Staiger, D. (2015). Technology diffusion and productivity growth in health care. The Review of Economics and Statistics, 97(5), 951-964.DOI: https://doi.org/10.1162/ REST_a_00535

Slovic, P. (1987). Perception of risk. Science, 236(4799), 280-285. DOI: https://doi.org/10.1126/science.3563507

Stiglic, M.; Agatz, N.; Savelsbergh, M.; Gradisar, M. (2016). Making dynamic ride-sharing work: the impact of driver and rider flexibility. Transportation Research Part E: Logistics and Transportation Review, 91, 190-207. DOI: https://doi. org/10.1016/j.tre.2016.04.010

Topham, G. (13 de octubre de 2017). Uber launches appeal against loss of London licence. The Guardian. Recuperado de https://bit.ly/3b7xc3R

White, D. R.; Burton, M. L.; Dow, M. M. (1981). Sexual division of labor in african agriculture: a network autocorrelation analysis. American Anthropologist, 83(4), 824-849. DOI: https://doi.org/10.1525/aa.1981.83.4.02a00040

Wittink, D. R.; Cattin, P. (1989). Commercial use analysis: an of conjoint update. Journal of Marketing, 53(3), 91-96. DOI: https://doi.org/10.2307/1251345

Wosskow, D. (2014). Unlocking the sharing economy: an independent review. Department for Business, Innovation and Skills, UK Government. Recuperado de https://bit.ly/3tunRJR 